\title{
Relación entre la dimensión del Parque Automotor con la Contaminación Acústica en la ciudad de Chota
}

Relation ship between the size of Automotive Park with noise pollution in the city of Chota

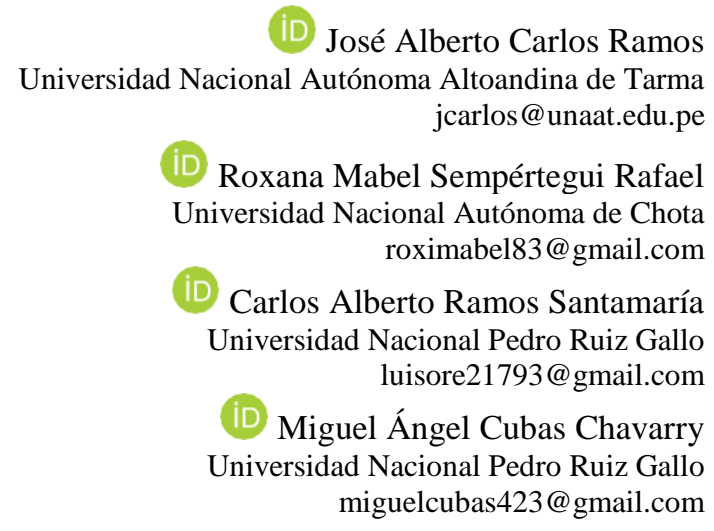

\section{RESUMEN}

Se midió el grado de ruido (dBA) ocasionado por el parque automotor de la ciudad de Chota, Región Cajamarca, aproximado en 3500 vehículos para un periodo de 6 meses. Se seleccionaron 12 puntos de muestreo teniendo en cuenta el mayor flujo vehicular. El grado de ruido se evaluó siguiendo los lineamientos del protocolo "Mediciones de ruido en exteriores" Se usó un sonómetro marca EXTECH, con rango desde 40 hasta $130 \mathrm{dBA}$, la frecuencia vehicular se evaluó en tres turnos, mañana $(7: 30 \mathrm{am})$, tarde $(12: 30 \mathrm{pm})$ y noche (7:00pm), por 15min". Se concluyó: El grado de ruido promedio de los 12 puntos monitoreados están en relación directa con la frecuencia vehicular y el uso inadecuado del claxon. El mapa de ruido permite definir puntos críticos con mayor notoriedad en la estación 5 que tiene un promedio de 83,82 dBA. Los niveles de ruido de las 12 estaciones monitoreadas variaron desde 70.14hasta 83.82dBA. Aun cuando los pobladores están acostumbrados a niveles elevados de ruido provocados por el parque automotor, se infiere que la calidad de vida es afectada por este problema ambiental.

Palabras clave: ruido, contaminación acústica, sonido. 


\begin{abstract}
The noise level (dBA) caused by the vehicle fleet in the city of Chota, Cajamarca Region, was measured, approximately 3500 vehicles for a period of 6 months. Twelve sampling points were selected taking into account the highest vehicular flow. The noise level was evaluated following the guidelines of the protocol "Outdoor noise measurements". An EXTECH sound level meter was used, with a range from 40 to $130 \mathrm{dBA}$, the vehicle frequency was evaluated in three shifts, morning (7:30am), afternoon (12:30pm) and night (7:00pm), for 15 minutes. It was concluded: The average noise level of the 12 monitored points is directly related to the frequency of vehicles and the inappropriate use of the horn. The noise map allows defining critical points with greater notoriety at station 5, which has an average of $83.82 \mathrm{dBA}$. The noise levels of the 12 monitored stations varied from 70.14 to $83.82 \mathrm{dBA}$. Even though the inhabitants are used to high noise levels caused by the vehicle fleet, it is inferred that the quality of life is affected by this environmental problem.
\end{abstract}

Keywords: noise, noise pollution, sound.

\section{INTRODUCCIÓN}

La contaminación ambiental que se evidencia en la actualidad, es producto de una deficiente política de desarrollo de la sociedad "en la que, la industrialización, marcó un punto de quiebre en la salud ambiental."

Se ha identificado en este contexto que el ambiente en el que se da la vida, es contaminado con agentes biológicos, químicos y físicos, se ha identificado al ruido como un agente físico que contamina al ambiente, produciendo la contaminación sonora.

Azquetay y Barry (1998), lo definen como "Una forma de energía mecánica que lleva asociada un flujo de energía, a la hora de medir la intensidad sonora (la velocidad con que dicha energía atraviesa una unidad de superficie, perpendicular a la dirección de propagación de la onda), se emplea una escala logarítmica, utilizando como unidad de medida el decibelio (dBA)". El ruido se da en grandes diferencias con respecto a otros contaminantes, por aparentar ser el más inofensivo, sin dejar residuos, pero, puede tener consecuencias acumulativas en el hombre.

El ruido ambiental, "Es el ruido asociado a un ambiente determinado y suele estar compuesto de sonidos de muchas fuentes, próximas y lejanas, sin un sonido dominante particular" (Bishop, 1993).

"Los niveles de ruido ambiental están creciendo en forma desproporcionada en todas las ciudades del mundo y sólo en España se calcula que al menos nueve millones de personas soportan niveles medios de 65 dBA, siendo el segundo país, detrás de Japón, con mayor índice de población expuesta a altos niveles de contaminación acústica” (Calle, 1999). 
(Pastor, 2005), "menciona que, durante los últimos cincuenta años, el aumento demográfico y el desarrollo industrial, se han generado acompañados de un aumento del nivel de ruido en las ciudades, una fuente importante, es el parque automotor. Los niveles del ruido por el parque automotor están en función de diversos parámetros tales como: tipo de vehículo, intensidad del tráfico vehicular, carga transportada, condiciones de utilización, estado de las pistas".

\section{METODOLOGÍA}

La metodología está siempre en función de los objetivos de la medición y de las condiciones en que la misma deberá llevarse a cabo Miyara (2000).

\section{La metodología que se empleó es la siguiente:}

Población:

"Áreas de contaminación acústica generadas por el parque automotor en la ciudad de Chota".

Muestra:

Nivel de ruido medido en cada estación según turno.

Unidad de análisis:

"Los resultados de las mediciones de los niveles de ruido y ruido ambiental se expresaron como el Nivel Equivalente de Presión Sonora Día, en decibelios A (dBA)"- Lento.

\section{Identificación de los puntos de monitoreo}

Identificamos 12 puntos de monitoreo teniendo en cuenta la mayor circulación vehicular; los cuales tienen que estar ubicados en las intersecciones de las principales calles y/o avenidas de la ciudad de Chota, los que se indican en la tabla 1 considerando la ubicación de las zonas colindantes

\section{Tabla 1}

\section{Puntos de monitoreo}

\begin{tabular}{|c|c|c|c|}
\hline Código & Referencia & Intersección & Zona \\
\hline E-1 & $\begin{array}{l}\text { Paradero a } \\
\text { Tacabamba }\end{array}$ & $\begin{array}{l}\text { Jr. Adriano Novoa. } \\
\text { Jr. Santa Rosa. }\end{array}$ & Comercial. \\
\hline E-2 & $\begin{array}{l}\text { Mercado nuevo } \\
\text { (Esquina) }\end{array}$ & $\begin{array}{l}\text { Jr. San Martin } \\
\text { Av. Todos los } \\
\text { santos. }\end{array}$ & Comercial. \\
\hline E-3 & $\begin{array}{l}\text { Paradero } \\
\text { Chuyabamba }\end{array}$ & $\begin{array}{l}\text { Av. Todos Los } \\
\text { Santos }\end{array}$ & Comercial. \\
\hline E-4 & Mercado central & Jr. Inca Garcilaso & Comercial. \\
\hline E-5 & $\begin{array}{l}\text { Paradero a } \\
\text { Chiclayo }\end{array}$ & $\begin{array}{l}\text { Av. José Osores } \\
\text { Jr. } 30 \text { de agosto }\end{array}$ & Comercial. \\
\hline E-6 & $\begin{array}{l}\text { Paradero a } \\
\text { Bambamarca }\end{array}$ & $\begin{array}{l}\text { Jr.: Mariscal } \\
\text { Castillo } \\
\text { Jr. Jose Arana } \\
\end{array}$ & Comercial. \\
\hline E-7 & $\begin{array}{l}\text { Una cuadra de la } \\
\text { plaza de armas }\end{array}$ & $\begin{array}{l}\text { Av. José Osores } \\
\text { Jr. } 30 \text { de agosto }\end{array}$ & Comercial. \\
\hline E-8 & Plaza de armas. & $\begin{array}{l}\text { Jr. Ponciano Vigil } \\
\text { Jr. } 30 \text { De Agosto }\end{array}$ & Comercial. \\
\hline E-9 & $\begin{array}{l}\text { Tiendas } \\
\text { Chancafe. }\end{array}$ & $\begin{array}{l}\text { Jr. Ponciano Vigil } \\
\text { Av. Inca Garcilaso }\end{array}$ & Comercial \\
\hline E-10 & Grifo Burga & Av. Inca Garcilaso & Terminal. \\
\hline E-11 & $\begin{array}{l}\text { Mercado Nuevo } \\
\text { (interior) }\end{array}$ & - & Comercial. \\
\hline E-12 & UNACH & $\begin{array}{l}\text { Campus } \\
\text { Universitario } \\
\text { (Colpa Matara) }\end{array}$ & $\begin{array}{l}\text { Construc- } \\
\text { ción y } \\
\text { educativa }\end{array}$ \\
\hline
\end{tabular}

Toma de mediciones en las estaciones de monitoreo Ubicados los puntos de monitoreo y utilizando 1 sonómetro EXTECH modelo 407727 se procedió a medir los niveles de ruido durante 15 minutos, en las horas de mayor circulación vehicular: 7:00 pm, 12:30 pm y 7:30 am, por 7 días. 


\section{RESULTADOS}

\section{Medición de los niveles de ruido}

En la tabla 2 se muestran los valores promedio totales de las 12 estaciones de monitoreo, en éste se puede observar lo siguiente:

Los niveles de ruido promedio varían de acuerdo al tiempo en que se toma las medidas del muestreo ya sea en las tardes, mañanas y noches respectivamente.
Los niveles máximos de ruido fueron registrados en un rango desde 83.42 hasta 90,91 dBA, los cuales se presentaron en las estaciones Av Todos los Santos intersección con el Jr San Martin _ Av. José Osores con la intersección de 30 de agosto, respectivamente. Los niveles mínimos de ruido fueron registrados desde 61,25 hasta $67,48 \mathrm{dBA}$, los cuales corresponden a las intersecciones Jr. Mariscal castilla, intersección inca Garcilaso de la vega, mercado central. Colpa Matara campus universitario

\section{Tabla 2}

Valores promedios

\begin{tabular}{|c|c|c|c|c|}
\hline Turno & Turno & Promedio (dB A) & $\begin{array}{c}\text { Nivel máximo } \\
\text { Según DS } 0665-20003 \text { PCM (dB A) }\end{array}$ & $\begin{array}{l}\text { Frecuencia } \\
\text { Vehicular }\end{array}$ \\
\hline \multirow{3}{*}{ E-1 } & Mañana & 75.4 & 70 & 80 \\
\hline & Tarde & 81.74 & 70 & 90 \\
\hline & Noche & 75.14 & 70 & 72 \\
\hline \multirow{3}{*}{ E-2 } & Mañana & 75.59 & 60 & 72 \\
\hline & Tarde & 83.42 & 60 & 98 \\
\hline & Noche & 74.34 & 60 & 70 \\
\hline \multirow{3}{*}{ E-3 } & Mañana & 75.52 & 70 & 75 \\
\hline & Tarde & 79.38 & 70 & 80 \\
\hline & Noche & 73.06 & 70 & 70 \\
\hline \multirow{3}{*}{ E-4 } & Mañana & 61.25 & 60 & 70 \\
\hline & Tarde & 78.95 & 60 & 83 \\
\hline & Noche & 70.23 & 60 & 70 \\
\hline \multirow{3}{*}{ E-5 } & Mañana & 82.37 & 70 & 89 \\
\hline & Tarde & 87.43 & 70 & 88 \\
\hline & Noche & 81.66 & 70 & 87 \\
\hline \multirow{3}{*}{ E-6 } & Mañana & 76.95 & 70 & 85 \\
\hline & Tarde & 80.97 & 70 & 95 \\
\hline & Noche & 72.80 & 70 & 80 \\
\hline \multirow{3}{*}{ E-7 } & Mañana & 71.5 & 70 & 80 \\
\hline & Tarde & 90.91 & 70 & 100 \\
\hline & Noche & 70.73 & 70 & 80 \\
\hline \multirow{3}{*}{ E-8 } & Mañana & 75.13 & 70 & 82 \\
\hline & Tarde & 78.79 & 70 & 90 \\
\hline & Noche & 69.55 & 70 & 78 \\
\hline \multirow{3}{*}{ E-9 } & Mañana & 75.48 & 60 & 88 \\
\hline & Tarde & 79.49 & 60 & 90 \\
\hline & Noche & 72.98 & 60 & 76 \\
\hline \multirow{3}{*}{ E-10 } & Mañana & 76.83 & 60 & 88 \\
\hline & Tarde & 78.29 & 60 & 90 \\
\hline & Noche & 72.32 & 60 & 77 \\
\hline \multirow{3}{*}{ E-11 } & Mañana & 74.16 & 60 & 82 \\
\hline & Tarde & 77.5 & 60 & 86 \\
\hline & Noche & 68.3 & 60 & 74 \\
\hline \multirow{3}{*}{ E-12 } & Mañana & 67.48 & 50 & 50 \\
\hline & Tarde & 77.66 & 50 & 56 \\
\hline & Noche & 66 & 50 & 45 \\
\hline
\end{tabular}




\section{DISCUSIÓN}

Los resultados obtenidos de la investigación tienen gran importancia para la sociedad, considerando que éstos serán utilizados para mitigar el impacto ambiental que genera el ruido producto del parque automotor de la ciudad de Chota sobre el ser humano, estas medidas de mitigación en proyectos ambientales que deberán implementarse a corto, mediano y largo plazo con políticas de desarrollo y que sean sostenibles(Méndez,2017)“y evitar mayores daños debido al aumento demográfico y tránsito vehicular". Los niveles de ruido del parque automotor en promedio que se reporta en la tabla 2 muestra que se debe aplicar una Ordenanza Municipal en las zonas comerciales "( $85 \mathrm{dBA})$, de las estaciones ubicadas en zona comercial", para ruidos en la ciudad de Chota no son tan rigurosas como las normas nacionales o internacionales, "mencionan que el ruido, por encima de los 50 dBA genera en el ser humano trastornos patológicos directos" tales como sordera, mayor incidencia de enfermedades cardiovasculares, digestivas y neurológicas, detectadas en personas expuestas a mayores niveles de ruido. "De acuerdo con la OMS, el nivel de sonido equivalente de las 8 horas de sueño no debería ser superior a $30 \mathrm{dBA}$ y en eventos únicos de ruido no deben exceder los 45 dBA"

La "perturbación del sueño que supone la presencia de ruido impide, asimismo que el cuerpo se recupere de la fatiga física y mental, con los efectos negativos" correspondientes. Además se comprobó que en las tardes hay mayor promedio de ruido en donde se registró más alta Av. José Osores con intersección del
Jr. 30 de agosto puesto a que este turno hay más circulación de vehículos en la ciudad chotana, se atribuye este resultado a que ésta es una zona marcada confluencia vehicular, puesto que por allí ingresan vehículos al centro de la población y hay una mayor circulación de moto taxis igualmente "es zona de salida en sentido inverso, además, es una vía de elevado tráfico en sentido" horizontal .

"Valores de elevada frecuencia vehicular se registraron también en la esquina" Av. Todos los Santos intersección con el Jr. San Martin, puesto que "son zonas de gran tráfico vehicular y porque el registro se realizó en horas punta, además dichas intersecciones tienen 3 ingresos de vehículos, en su mayoría. Vehículos de servicio público como combis," mototaxis, colectivos y cousters por el mismo hecho que en esa zona se encuentra el mercado mayorista donde todo esto genera una contaminación acústica muy significativa con elevados niveles de ruido.

Respecto "a los estándares de calidad ambiental para ruido a nivel nacional (D.S. $\mathrm{N}^{\circ}$ 085-2003-PCM) e internacional OMS (1993) los niveles de ruido hallados en las 10 estaciones superaron los LMP de estas normas. En los casos de las estaciones consideradas zonas" comerciales como mercado, paraderos entre otras superan "la norma (70 dBA) las cifras del exceso varían desde 7hasta 11 y 13,36 dBA. Esto es de especial interés para nuestro estudio por cuanto el incremento del ruido se hace de forma logarítmica; es decir, el aumente de 1 dBA significa el aumento de 100 veces el nivel de ruido tal como lo menciona" Miyara (2000). 
Al respecto Chávez (s/f) "menciona que ello genera la posibilidad de que los transeúntes o pobladores se afecten con el denominado Trauma Acústico Crónico (TAC) o Daño Auditivo Inducido por Ruido (DAIR), produciéndose un déficit o caída inicial generalmente entre los 4000 y 6000 Hz."

El mismo autor "menciona que existen otros efectos atribuidos a la exposición al ruido, tales como la Desviación Transitoria del Umbral de la Audición, que se caracteriza por una ligera disminución de la sensibilidad auditiva, son transitoria, dura poco tiempo y no suele sobrepasar las 16 horas. El Trauma Acústico Agudo que se produce como consecuencia del efecto abrupto del ruido y que lo más frecuente que suceda es una sordera de tipo conductiva por lesión de las estructuras del oído externo" y medio. Se explica estos resultados "por el hecho que en las vías aledañas se registró alta frecuencia vehicular y la medición se tomó en horas punta; resultados" que son compatibles con lo expresado por Sichez (2000), reporta que "en la Población de la Ciudad de Trujillo, el nivel de ruido fue mayor a $85 \mathrm{dBA}$, y señala que este efecto disminuye significativamente el bienestar" de la población.

En el turno mañana Jr. mariscal castilla, intersección con el Jr José Arana “presentó el mayor nivel de ruido promedio con $76.95 \mathrm{dBA}$, seguido por la estación de Jr. inca Garcilaso de la vega y el menor nivel de ruido" fue en Jr. Mariscal castilla, intersección inca Garcilaso de la vega, mercado central. con un promedio de $61.25 \mathrm{dBA}$.

En el turno tarde, la estación de la Av. José Osores intersección con 30 de agosto "presentó el más alto nivel de ruido con $90.25 \mathrm{dBA}$, siendo esta zona de protección especial, la situación es preocupante, ya que en esa zona se ubican donde hay centros educativos Las estaciones de menor nivel de ruido son" Av. Todos los Santos intersección con el Jr San Martin (interior del mercado) con un rango de 77.50dBA, "se atribuyen estos resultados a la menor frecuencia" de peatones.

En "el turno de noche el nivel más alto de ruido estuvo" en Jr Adriano Novoa, intersección con el Jr santa rosa con $75.14 \mathrm{dBA}$, por ser paradero y el menor nivel de ruido en ese turno fue Jr Ponciano Vigil, intersección con el Jr 30 de agosto con un promedio de 69.55 .

Al "analizar los niveles de ruido medidos por turno, podemos inferir que no hay mayores diferencias, aspecto que se reporta por primera vez para la ciudad" de Chota.

La encuesta, como una "escala tipo Likert, permitió medir los conocimientos para generar cambios de actitudes y comportamientos en el poblador chotano respecto a la contaminación acústica y su relación con el parque" automotor.

Los "pobladores, en términos genéricos, desconocen si el nivel de ruido generado por el parque automotor es igual en toda la ciudad; se explica esta respuesta por el desconocimiento que tienen respecto a este contaminante ambiental; sin embargo consideramos importante sugerir a las autoridades municipales y entidades comprometidas con la salud de los pobladores para desarrollar campañas de instrucción sobre el nocivo impacto que se genera e implementar medidas de medidas de mitigación y evitar daños en la salud" humana.

El "Ítem con más alto puntaje (90): se debe educar a los conductores para disminuir la contaminación acústica en Chota, refleja un nivel de conocimientos 
aceptable al identificar claramente a quienes generan el ruido, los choferes o conductores; al mismo tiempo se aprecia una actitud preventiva, proactiva y de protección para instruir a los generadores de la contaminación acústica, y proteger la salud humana; ello coincide con los criterios de Stanfeld citados" por Pastor (2005) "ya que los picos más altos de nivel de ruido fueron ocasionados por el uso indiscriminado de claxon de las camionetas rurales, taxis o vehículos pesados. En algunos casos se colocan claxon de vehículos pesados en las camionetas rurales provocando un mayor nivel de ruido por las zonas" en las que circulan.

Se identificó que el uso de claxon "se dio por tres motivos: i) los taxis usan el claxon para motivar a los usuarios o pasajeros, ii) los vehículos usan el claxon para apurar a vehículos que transitan delante. Un comportamiento típico se da cuando las luces del semáforo cambian en las intersecciones, o cuando un vehículo se detiene delante de otro; iii) Los vehículos usan el claxon en situaciones conflictivas lo que se ha convertido en una mala práctica en la ciudad, en el momento que los conductores, en vez de dar paso a otros vehículos o peatones, hacen sonar el claxon como una manera de hacer notar la presencia" de su unidad móvil.

\section{CONCLUSIONES}

El grado de ruido promedio de los 12 puntos monitoreados están en relación directa con la frecuencia vehicular y el uso inadecuado del claxon.
El mapa de ruido permite definir puntos críticos con mayor notoriedad en la estación 5 que tiene un promedio de $83,82 \mathrm{dBA}$.

Los niveles de ruido de las 12 estaciones monitoreadas variaron desde 70.14 hasta $83.82 \mathrm{dBA}$. Aun cuando los pobladores están acostumbrados a niveles elevados de ruido provocados por el parque automotor, se infiere que la calidad de vida es afectada por este problema ambiental.

\section{REFERENCIAS}

[1] Azqueta, D. y F. Barry. (1998). Economía y Medio Ambiente.Tomo 3. McGraw-Hill. 2a . Edición. Bogotá-Colombia.

[2] Bishop, D. (1993). Program for the measurement of environmental noise. Department of Transportation. Washington, D. C.

[3] Calle, M. (1999). Contaminación acústica y salud.

http://www.sorolls.org/docs/Contaminacion _acustica_WASTE_magazine.htm

[4] Chavez, J. S/F. Trauma Acústico Crônico. MedSalud

S.A.C http://www.preriesgo.com/boletin8/articulo2 .htm

[5] Méndez, M. (2017). Parque automotor y contaminación ambiental en el Centro Histórico de Lima (Doctoral dissertation), Tesis de pregrado, Universidad Nacional Mayor de San Marcos,lima Perú).http://cybertesis.unmsm.edu.pe/handle $/ 20.500 .12672 / 6200$ 
[6] Miyara, F. (2000). Control de Ruido, Publicado dentro del libro en CD-ROM jornadas Internacionales Multidisciplinarias sobre Violencia Acústica. Editorial ASOLOFAL. Rosario. Argentina.

[7] Organización Mundial de la Salud. 1993. Guideline values for community noise. Organización Mundial de la Salud-OMS. Ginebra.

http://www.ruidos.org/Noise/WHO_Noise_ guidelines_4.html

[8] Pastor, J. (2005). Efectos de la contaminación acústica sobre la capacidad auditiva de los pobladores de la ciudad de Trujillo-Perú. Tesis para optar el grado de Doctor en Medio Ambiente. Universidad Nacional de Trujillo. Perú.

[9] Sichez, J. (2000). Contaminación sonora e impactos en el bienestar de la población de Trujillo 1999. Tesis para optar el Grado Académico de Maestro en Ciencias con mención en Gestión Ambiental. Universidad Nacional de Trujillo. Perú. 